\title{
Impact of the Preoperative C-reactive Protein to Albumin Ratio on the Long-Term Outcomes of Hepatic Resection for Intrahepatic Cholangiocarcinoma
}

\author{
Tokuji Ito*, Hiroji Shinkawa, Shigekazu Takemura, Shogo Tanaka, Takayoshi \\ Nishioka, Toru Miyazaki, Atsushi Ishihara, Shoji Kubo
}

\begin{abstract}
Objective: The present study aimed to investigate the impact of preoperative C-reactive protein to albumin (CRP/Alb) ratio on the long-term outcomes of patients with intrahepatic cholangiocarcinoma (ICC). Methods: 82 patients who underwent hepatic resection for mass-forming type of ICC were evaluated. The relationship between preoperative $\mathrm{CRP} / \mathrm{Alb}$ ratio and survival outcomes was investigated. Results: The optimal cutoff value of CRP/Alb ratio for assessing overall survival (OS) was determined as 0.089 . Univariate analysis for recurrence-free survival (RFS) showed that $\mathrm{CRP} / \mathrm{Alb}$ ratio $>0.089$, carbohydrate antigen 19-9 (CA 19-9) $>37 \mathrm{U} / \mathrm{mL}$, lymph node metastasis, vascular invasion, and multiple tumors were significantly associated with postoperative recurrence. On multivariate analysis, the independent prognostic factors identified were CRP/Alb ratio $>0.089(p<0.001)$, lymph node metastasis $(p=0.006)$, and multiple tumors $(\mathrm{p}<0.001)$. Univariate analysis for OS showed that CRP/Alb ratio $>0.089, \mathrm{CA} 19-9>37 \mathrm{U} / \mathrm{mL}$, lymph node metastasis, vascular invasion, multiple tumors, and positive surgical margin were significantly associated with overall death. On multivariate analysis, the independent prognostic factors identified were CRP/Alb ratio $>0.089$ $(p<0.001)$, lymph node metastasis $(p=0.01)$, and multiple tumors $(p=0.005)$. Conclusion: Preoperative CRP/Alb ratio may predict poor long-term outcomes after hepatic resection in patients with ICC.
\end{abstract}

Keywords: CRP/Alb ratio- intrahepatic cholangiocarcinoma- hepatic resection- prognostic factor

Asian Pac J Cancer Prev, 21 (8), 2373-2379

\section{Introduction}

Intrahepatic cholangiocarcinoma (ICC), which arises from the bile ducts in the liver, is the second most common form of primary hepatic cancer after hepatocellular carcinoma (Shirabe et al., 2010; Farges et al., 2011; Zou et al., 2014; Siegel et al., 2015; Bridgewater et al., 2014). The incidence of ICC in East Asia is the highest in the world; however, recent nationwide population-based studies have shown an increasing incidence of ICC in western countries (Petrick et al., 2016). Currently, surgery is the only potentially curative treatment option for ICC. Despite the recent advances in surgical techniques, chemotherapy, and radiotherapy, the survival outcome after surgery remains poor, with a five-year survival rate of approximately $30 \%$ (Farges et al., 2010). Therefore, useful markers for stratifying the prognosis of patients with ICC are needed to develop appropriate treatment strategies that can improve survival outcomes after surgery.

Recently, there has been increasing evidence that systemic inflammatory response predicts the survival outcome in cancer patients. C-reactive protein (CRP), which is one of the inflammatory markers, is an acute-phase reactant that is synthesized by hepatocytes (Morris-Stiff et al., 2008; Nakazaki, 1992). Previous studies have demonstrated that an increased level of CRP is associated with worse prognosis in patients with various kinds of tumors (Nozoe et al., 1998; Hashimoto et al., 2005; Pathak et al., 2014; Ishino et al., 2014; Shrotriya et al., 2015). Further, serum albumin (Alb), which is the traditional standard marker of nutritional status, is also known to reflect inflammatory status (Ishida et al., 2014). Combination of these inflammatory markers into a CRP/ Alb ratio had been demonstrated as a prognostic indicator in several kinds of cancers, such as colorectal (Ishizuka et al., 2016; Shibutani et al., 2016), gastric (Saito et al., 2018), esophageal (Wei et al., 2015), hepatocellular (Kinoshita et al., 2015), and pancreatic (Haruki et al., 2016; Liu et al., 2017). Therefore, we believe that the $\mathrm{CRP} / \mathrm{Alb}$ ratio may predict the postoperative prognosis of patients with ICC.

In this study, we retrospectively investigated the relationship between preoperative $\mathrm{CRP} / \mathrm{Alb}$ ratio and survival outcomes in patients who underwent hepatic 
resection for ICC.

\section{Materials and Methods}

\section{Patients}

The medical records of 82 patients who underwent initial and curative hepatic resection for a mass-forming (MF) type of ICC at the Osaka City University Hospital between January 1998 and October 2017 were retrospectively reviewed. In this study, ICC was pathologically defined as cholangiocarcinoma centering in second or more peripheral branches of the intrahepatic bile ducts. There were no patients with jaundince, cholangitis or bile duct drainage. ICC tumors were classified according to the macroscopic classification proposed by the Liver Cancer Study Group of Japan (Liver Cancer Study Group of Japan, 2010). Patients with pure intraductal growth or pure periductal-infiltrating type of ICC were excluded because these types of ICC were known to have different biological behaviors from that of MF-dominant ICC (Shimada et al., 2007; Yamamoto et al., 2009; Uno et al., 2012). Curative resection was defined as the complete removal of the entire macroscopic tumor without residual tumors. All analyses were performed in accordance with the ethical guidelines for clinical studies at Osaka City University Hospital (approval number, 3815). Comprehensive informed consent to use patient information for this study was obtained from the patients before surgery.

\section{Clinical data extraction}

We collected the baseline characteristic of the participants, including age, gender, and disease stage, which was determined based on the Eighth Edition of the American Joint Committee on Cancer staging criteria. All blood test values recorded in this study were obtained within 2-4 days before the operation and included CRP, Alb, alanine aminotransferase (ALT), carbohydrate antigen 19-9 (CA 19-9), and carcinoembryonic antigen (CEA). The CRP/Alb ratio was calculated by dividing the serum CRP level by the serum Alb level.

\section{Patient follow-up}

After hepatic resection, all patients were regularly screened for recurrence by monitoring the plasma levels of tumor markers (CEA and CA 19-9), ultrasonography, and dynamic computed tomography. Recurrence was defined as the appearance of a new tumor lesion that had radiological features of ICC. When recurrence was detected, the patient received further treatment like repeated hepatic resection or others, such as chemotherapy. The period of follow-up was from the date of the initial surgery until March 30, 2018 or death from any cause. Patients who did not die at the time of last follow-up were censored.

\section{Statistical analysis}

All statistical analyses were performed using EZR software (Saitama Medical Center, Jichi Medical University, Saitama, Japan), which is the graphical user interface for R (The R Foundation for Statistical Computing, Vienna, Austria) (Kanda, 2013). Categorical variables were compared using the Fisher exact probability method or $\chi^{2}$ test. Continuous variables were presented as median (range). The Mann-Whitney U-test was performed to evaluate the differences between the two groups. The optimal cutoff level of the CRP/Alb ratio was determined by a web-based system that was engineered by the $R$ software (http://molpath.charite.de/cutoff) (Budczies et al., 2012). Survival curves were calculated according to the Kaplan-Meier method. Univariate analyses were conducted using the log-rank test. Multivariate analyses of the variables that affected survival were performed using the Cox proportional hazards model. P-values were derived from two-tailed tests, and statistical significance was set at $<0.05$.

The variables that were potentially associated with postoperative prognosis were selected based on the results of a previous study and on our own clinical experience (Uenishi et al., 2014; Spolverato et al., 2015; Pan et al., 2017). These included age ( $\leq 65$ or $>65$ years); sex; body mass index ( $\leq 25$ or $>25 \mathrm{~kg} / \mathrm{m} 2)$; hepatitis virus infection; CRP $(\leq 0.4$ or $>0.4 \mathrm{mg} / \mathrm{dL})$; Alb concentration $(\leq 4.0$ or $>4.0 \mathrm{~g} / \mathrm{dL})$; ALT activity $(\leq 30$ or $>30 \mathrm{IU} / \mathrm{L})$; liver cirrhosis, CEA ( $\leq 5$ or $>5 \mathrm{ng} / \mathrm{mL})$; CA $19-9$ ( $\leq 37$ or $>37$ $\mathrm{U} / \mathrm{mL})$; largest diameter of the main tumor $(>5.0$ or $\leq 5.0$ $\mathrm{cm}$ ); single or multiple tumors; presence of intrahepatic metastases, lymph node metastasis, bile duct tumor invasion, or microscopic vascular invasion; surgical margin; and adjuvant chemotherapy.

\section{Results}

\section{Clinicopathological profiles of the patients}

The clinicopathological characteristics of the patients are detailed in Supplementary Table 1. The number of patients classified as stage I, II, III, and IVa were 21, 23,15 , and 23, respectively. Of all patients, 29 (35.4\%) had hepatitis virus infection, and $9(11 \%)$ had alcoholic hepatitis. The overall median follow-up duration was 43.1 months (range, 2-127.5 months). During the study period, 50 patients $(61 \%)$ had postoperative recurrence, and 45 patients $(54.9 \%)$ died.

\section{Cutoff value of the CRP/Alb ratio}

Using the biostatistical tool, Cutoff Finder, we found a wide range of cutoff values for the CRP/Alb ratio and determined 0.089 as the optimal value for assessing overall survival (OS) (Figure. 1). Patients were divided into two groups based on the cutoff value of the $\mathrm{CRP} / \mathrm{Alb}$, as follows: the $\mathrm{CRP} / \mathrm{Alb}$ ratio $>0.089$ group $(\mathrm{n}=26)$ and the $\mathrm{CRP} / \mathrm{Alb}$ ratio $\leq 0.089$ group $(\mathrm{n}=56)$.

Relationship between the preoperative CRP/Alb ratio and the clinicopathological features of patients with ICC

Table 1 shows the clinicopathological characteristics of the patients based on the CRP/Alb ratio. Compared with the $\mathrm{CRP} / \mathrm{Alb}$ ratio $\leq 0.089$ group, the $\mathrm{CRP} / \mathrm{Alb}$ ratio $>0.089$ group comprised significantly more patients with tumor size $>5 \mathrm{~cm}(50 \%$ vs. $21.4 \%, \mathrm{p}=0.019)$ and bile duct tumor invasion ( $65.4 \%$ vs. $39.3 \%, \mathrm{p}=0.034)$. Microvascular invasion tended to be frequently observed in in the $\mathrm{CRP} / \mathrm{Alb}>0.089$ group than the $\leq 0.089$ group $(61.5 \%$ vs. $37.5 \%, p=0.057)$. 
DOI:10.31557/APJCP.2020.21.8.2373

CRP/Alb Ratio for Intrahepatic Cholangiocarcinoma

Table 1. Comparison of Patients with Intrahepatic Cholangiocarcinoma Categorized by C-Reactive Protein to Albumin Ratio

\begin{tabular}{|c|c|c|c|}
\hline Variable & $\begin{array}{c}\text { CRP/Alb } \\
>0.089 \\
(n=26)\end{array}$ & $\begin{array}{c}\mathrm{CRP} / \mathrm{Alb} \\
\leq 0.089 \\
(\mathrm{n}=56)\end{array}$ & $\begin{array}{c}p- \\
\text { value }\end{array}$ \\
\hline Gender (Male/Female) & $18 / 8$ & $42 / 14$ & 0.6 \\
\hline Age (years) & $67.5(49-82)$ & $68(32-82)$ & 0.78 \\
\hline Age $>65$ (years) & $16(61.5 \%)$ & $32(57.1 \%)$ & 0.81 \\
\hline $\mathrm{BMI}>25$ & $8(30.8 \%)$ & $17(30.4 \%)$ & $>0.99$ \\
\hline Hepatitis Virus presence & $6(23.1 \%)$ & $23(41.2 \%)$ & 0.14 \\
\hline ALT $>30(\mathrm{IU} / \mathrm{L})$ & $10(38.5 \%)$ & $24(42.9 \%)$ & 0.81 \\
\hline $\mathrm{CEA}>5(\mathrm{ng} / \mathrm{mL})$ & $9(52.9 \%)$ & $17(30.3 \%)$ & 0.8 \\
\hline CA19-9 >37 (U/mL) & $14(53.8 \%)$ & $24(42.9 \%)$ & 0.48 \\
\hline Liver cirrhosis & $2(8.3 \%)$ & $9(16.1 \%)$ & 0.49 \\
\hline TNM classification (III-IVa) & $13(50 \%)$ & $25(44.6 \%)$ & 0.81 \\
\hline Lymph node metastasis & $5(23.8 \%)$ & $13(23.2 \%)$ & 0.78 \\
\hline Bile duct invasion & $17(65.4 \%)$ & $22(39.3 \%)$ & 0.034 \\
\hline Microvascular invasion & $16(61.5 \%)$ & $21(37.5 \%)$ & 0.057 \\
\hline Multiple tumor & $6(23.1 \%)$ & $13(23.2 \%)$ & $>0.99$ \\
\hline Tumor size >5 $(\mathrm{cm})$ & $13(50 \%)$ & $12(21.4 \%)$ & 0.019 \\
\hline Surgical margin positive & $5(23.8 \%)$ & $7(12.5 \%)$ & 0.51 \\
\hline Adjuvant chemotherapy & $10(38.5 \%)$ & $31(55.4 \%)$ & 0.24 \\
\hline
\end{tabular}

CRP/Alb, C-reactive protein/albumin; BMI, body mass index; ALT, alanine aminotransferase; CEA, carcinoembryonic antigen; CA19-9, carbohydrate antigen 19-9; TNM, tumor-node-metastasis

Table 2. Prognostic Factors for Recurrence-Free Survival

\begin{tabular}{|c|c|c|c|c|c|}
\hline \multirow[t]{2}{*}{ Variable } & \multirow[b]{2}{*}{$\mathrm{N}$} & \multicolumn{2}{|c|}{ Univariate } & \multicolumn{2}{|c|}{ Multivariate } \\
\hline & & HR $(95 \% \mathrm{CI})$ & p-value & $\operatorname{HR}(95 \% \mathrm{CI})$ & p-value \\
\hline \multicolumn{6}{|l|}{ Gender } \\
\hline Male & 60 & $0.59(0.32-1.07)$ & 0.083 & & \\
\hline Female & 22 & & & & \\
\hline \multicolumn{6}{|l|}{ Age (year) } \\
\hline$>65$ & 48 & $1.37(0.77-2.44)$ & 0.29 & & \\
\hline$\leq 65$ & 34 & & & & \\
\hline \multicolumn{6}{|c|}{ BMI $\left(\mathrm{kg} / \mathrm{m}^{2}\right)$} \\
\hline$>25$ & 25 & $1.05(0.58-1.89)$ & 0.88 & & \\
\hline$\leq 25$ & 57 & & & & \\
\hline \multicolumn{6}{|c|}{ Hepatitis virus infection } \\
\hline Presence & 29 & $1.17(0.65-2.08)$ & 0.6 & & \\
\hline Absence & 53 & & & & \\
\hline \multicolumn{6}{|c|}{$\mathrm{CRP}(\mathrm{mg} / \mathrm{dl})$} \\
\hline$>0.4$ & 21 & $1.39(0.72-2.69)$ & 0.32 & & \\
\hline$\leq 0.4$ & 61 & & & & \\
\hline \multicolumn{6}{|l|}{$\mathrm{Alb}(\mathrm{g} / \mathrm{dl})$} \\
\hline$>4$ & 41 & $1.40(0.80-2.46)$ & 0.24 & & \\
\hline$\leq 4$ & 41 & & & & \\
\hline \multicolumn{6}{|l|}{$\mathrm{CRP} / \mathrm{Alb}$} \\
\hline$>0.089$ & 26 & $2.24(1.25-4.01)$ & 0.007 & $3.00(1.58-5.69)$ & $<0.001$ \\
\hline$\leq 0.089$ & 56 & & & & \\
\hline \multicolumn{6}{|c|}{ ALT (IU/L) } \\
\hline$>30$ & 34 & $1.28(0.73-2.25)$ & 0.39 & & \\
\hline$\leq 30$ & 48 & & & & \\
\hline
\end{tabular}

Table 2. Continued

\begin{tabular}{|c|c|c|c|c|c|}
\hline \multirow[t]{2}{*}{ Variable } & \multirow[b]{2}{*}{$\mathrm{N}$} & \multicolumn{2}{|c|}{ Univariate } & \multicolumn{2}{|c|}{ Multivariate } \\
\hline & & HR $(95 \% \mathrm{CI})$ & p-value & $\mathrm{HR}(95 \% \mathrm{CI})$ & p-value \\
\hline \multicolumn{6}{|c|}{ Liver cirrhosis } \\
\hline Presence & 11 & $1.35(0.67-2.79)$ & 0.41 & & \\
\hline Absence & 71 & & & & \\
\hline \multicolumn{6}{|c|}{ CEA (ng/mL) } \\
\hline$>5$ & 26 & $1.27(0.70-2.31)$ & 0.43 & & \\
\hline$\leq 5$ & 56 & & & & \\
\hline \multicolumn{6}{|c|}{ CA19-9 (U/mL) } \\
\hline$>37$ & 38 & $1.81(1.03-3.20)$ & 0.039 & $1.04(0.55-1.99)$ & 0.9 \\
\hline$\leq 37$ & 44 & & & & \\
\hline \multicolumn{6}{|c|}{ Lymph node metastasis } \\
\hline Presence & 18 & $2.98(1.62-5.49)$ & $<0.001$ & $2.64(1.32-5.30)$ & 0.006 \\
\hline Absence & 64 & & & & \\
\hline \multicolumn{6}{|c|}{ Bile duct invasion } \\
\hline Presence & 49 & $1.58(0.90-2.78)$ & 0.11 & & \\
\hline Absence & 43 & & & & \\
\hline \multicolumn{6}{|c|}{ Vascular invasion } \\
\hline Presence & 37 & $2.13(1.21-3.74)$ & 0.009 & $1.42(0.78-2.60)$ & 0.25 \\
\hline Absence & 45 & & & & \\
\hline \multicolumn{6}{|c|}{ Tumor number } \\
\hline Multiple & 19 & $2.93(1.57-5.45)$ & $<0.001$ & $3.34(1.69-6.61)$ & $<0.001$ \\
\hline Single & 63 & & & & \\
\hline \multicolumn{6}{|c|}{ Tumor size $(\mathrm{cm})$} \\
\hline$>5$ & 25 & $1.70(0.94-3.05)$ & 0.77 & & \\
\hline$\leq 5$ & 57 & & & & \\
\hline \multicolumn{6}{|c|}{ Surgical margin } \\
\hline Positive & 12 & $1.63(0.79-3.37)$ & 0.19 & & \\
\hline Negative & 70 & & & & \\
\hline \multicolumn{6}{|c|}{ Adjuvant chemotherapy } \\
\hline Yes & 41 & $1.15(0.65-2.02)$ & 0.63 & & \\
\hline No & 41 & & & & \\
\hline
\end{tabular}

$\mathrm{HR}$, hazard ratio; $\mathrm{CI}$, confidence interval; $\mathrm{CRP} / \mathrm{Alb}$, C-reactive protein albumin; BMI, body mass index; ALT, alanine aminotransferase; CEA, carcinoembryonic antigen; CA19-9, carbohydrate antigen 19-9

\section{Recurrence-free survival (RFS) and OS}

The respective one-, three-, and five-year RFS rates after surgery were $39 \%, 17 \%$, and $17 \%$ in the CRP/ Alb ratio $>0.089$ group and $73 \%, 46 \%$, and $38 \%$ in the $\mathrm{CRP} / \mathrm{Alb}$ ratio $\leq 0.089$ group $(\mathrm{p}=0.007)$ (Figure 2$)$. The respective one-, three-, and five-year OS rates after surgery were $65 \%, 31 \%$, and $18 \%$ in the $\mathrm{CRP} / \mathrm{Alb}$ ratio $>0.089$ group and $90 \%, 66 \%$, and $48 \%$ in the $\mathrm{CRP} / \mathrm{Alb}$ ratio $\leq 0.089$ group $(\mathrm{p}<0.001)$ (Figure 3$)$.

\section{Prognostic factors for RFS among patients with ICC after hepatic resection}

Table 2 shows the prognostic factors for RFS. According to the univariate analysis, the $\mathrm{CRP} / \mathrm{Alb}$ ratio $>0.089$, CA19-9 >37, lymph node metastasis, vascular invasion, and multiple tumors were significantly associated with postoperative recurrence. The independent prognostic factors for RFS, according to the multivariate analysis, were $\mathrm{CRP} / \mathrm{Alb}$ ratio $>0.089$ [hazard ratio (HR), 3.00; 95\% confidence interval (CI), 1.58-5.69; $\mathrm{p}<0.001$ ]; lymph node metastasis (HR, 2.64; 95\% CI, 1.32-5.30; 


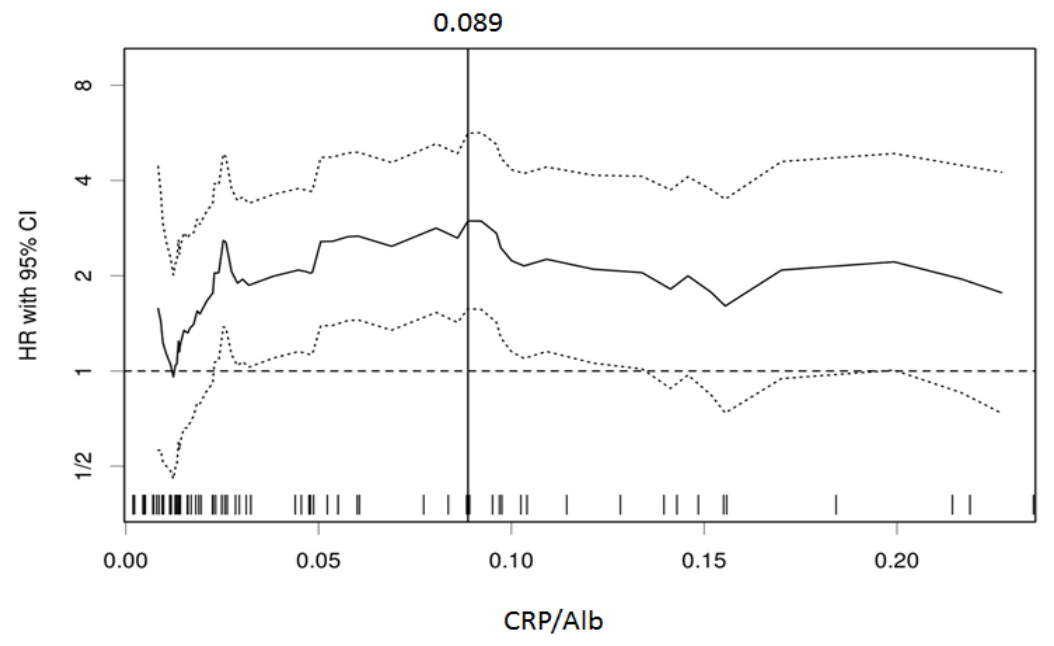

Figure 1. Association between Preoperative CRP/Alb Ratio and Overall Survival of Patients with Intrahepatic Cholangiocarcinoma. The hazard ratio (HR) including the $95 \%$ confidence interval (CI) is plotted in relation to the cutoff values. The vertical line designates the dichotomization showing the most significant correlation with overall survival.

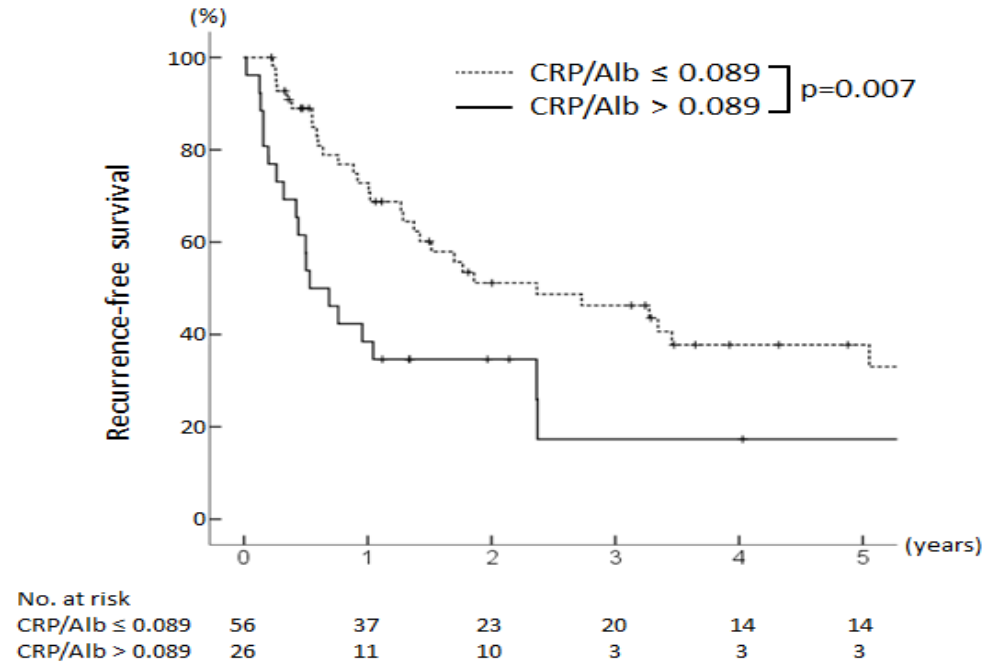

Figure 2. Kaplan-Meier Analysis of the Recurrence Free Survival of Intrahepatic Cholangiocarcinoma Patients with a High $(>0.089)$ vs Those with a Low $(\leq 0.089) \mathrm{CRP} /$ Alb Ratio. There were significant difference between the groups.

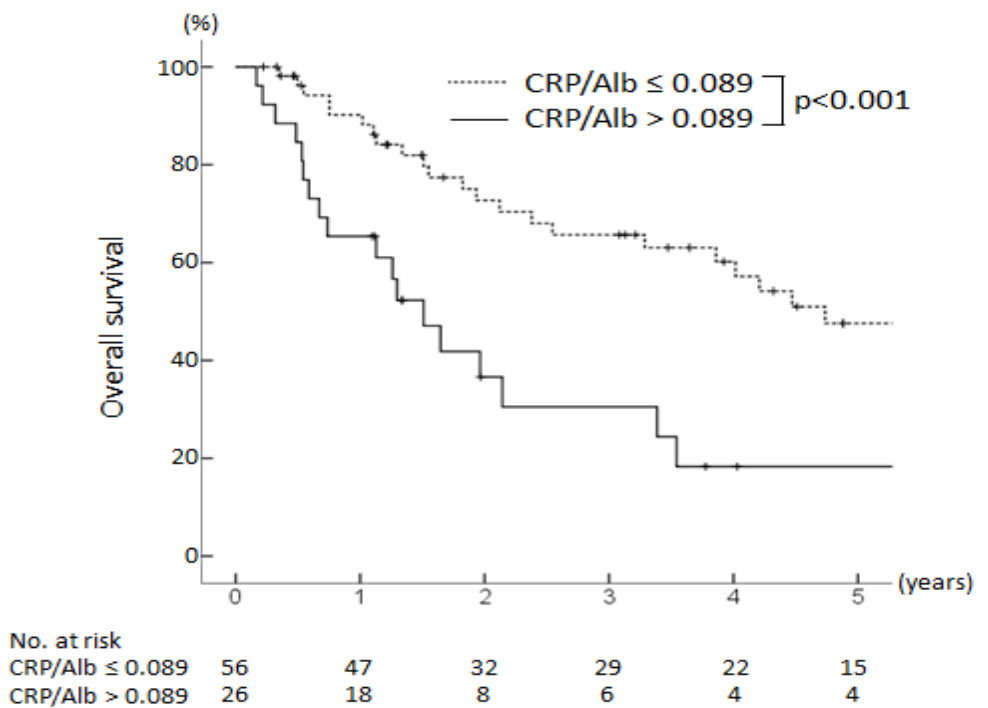

Figure 3. Kaplan-Meier Analysis of the Overall Survival of Intrahepatic Cholangiocarcinoma Patients with a High $(>0.089)$ vs Those with a Low $(\leq 0.089) \mathrm{CRP} / \mathrm{Alb}$ Ratio. There were significant difference between the groups. 
DOI: 10.31557/APJCP.2020.21.8.2373

CRP/Alb Ratio for Intrahepatic Cholangiocarcinoma

Table 3. Prognostic Factors for Overall Survival

\begin{tabular}{|c|c|c|c|c|c|}
\hline \multirow[t]{2}{*}{ Variable } & \multicolumn{3}{|c|}{ Univariate } & \multicolumn{2}{|l|}{ Multivariate } \\
\hline & $\mathrm{N}$ & HR (95\% CI) & $\mathrm{p}$-value & HR(95\% CI) & $\mathrm{p}$-value \\
\hline \multicolumn{6}{|l|}{ Gender } \\
\hline Male & 60 & $0.71(0.37-1.33)$ & 0.28 & & \\
\hline Female & 22 & & & & \\
\hline \multicolumn{6}{|l|}{ Age (year) } \\
\hline$>65$ & 48 & $1.27(0.70-2.32)$ & 0.43 & & \\
\hline$\leq 65$ & 34 & & & & \\
\hline \multicolumn{6}{|c|}{$\mathrm{BMI}\left(\mathrm{kg} / \mathrm{m}^{2}\right)$} \\
\hline$>25$ & 25 & $0.94(0.50-1.77)$ & 0.85 & & \\
\hline$\leq 25$ & 57 & & & & \\
\hline \multicolumn{6}{|c|}{ Hepatitis virus infection } \\
\hline Presence & 29 & $0.97(0.53-1.80)$ & 0.93 & & \\
\hline Absence & 53 & & & & \\
\hline \multicolumn{6}{|c|}{ CRP (mg/dl) } \\
\hline$>0.4$ & 21 & $1.52(0.77-3.03)$ & 0.23 & & \\
\hline$\leq 0.4$ & 61 & & & & \\
\hline \multicolumn{6}{|l|}{$\mathrm{Alb}(\mathrm{g} / \mathrm{dl})$} \\
\hline$>4$ & 41 & $0.84(0.84-2.74)$ & 0.17 & & \\
\hline$\leq 4$ & 41 & & & & \\
\hline \multicolumn{6}{|l|}{ CRP/Alb } \\
\hline$>0.089$ & 26 & $2.98(1.57-5.64)$ & $<0.001$ & $3.39(1.72-6.67)$ & $<0.001$ \\
\hline$\leq 0.089$ & 56 & & & & \\
\hline \multicolumn{6}{|l|}{ ALT (IU/L) } \\
\hline$>30$ & 34 & $1.65(0.92-2.96)$ & 0.095 & & \\
\hline$\leq 30$ & 48 & & & & \\
\hline \multicolumn{6}{|c|}{ Liver cirrhosis } \\
\hline Presence & 11 & $1.16(0.51-2.63)$ & 0.72 & & \\
\hline Absence & 71 & & & & \\
\hline \multicolumn{6}{|c|}{ CEA (ng/mL) } \\
\hline$>5$ & 26 & $1.20(0.62-2.30)$ & 0.59 & & \\
\hline$\leq 5$ & 56 & & & & \\
\hline \multicolumn{6}{|c|}{ CA19-9 (U/mL) } \\
\hline$>37$ & 38 & $1.83(1.01-3.31)$ & 0.047 & $1.20(0.60-2.40)$ & 0.61 \\
\hline$\leq 37$ & 44 & & & & \\
\hline \multicolumn{6}{|c|}{ Lymph node metastasis } \\
\hline Presence & 18 & $3.36(1.73-6.56)$ & $<0.001$ & $2.82(1.29-6.19)$ & 0.01 \\
\hline Absence & 64 & & & & \\
\hline \multicolumn{6}{|c|}{ Bile duct invasion } \\
\hline Presence & 49 & $1.42(0.78-2.57)$ & 0.25 & & \\
\hline Absence & 43 & & & & \\
\hline \multicolumn{6}{|c|}{ Vascular invasion } \\
\hline Presence & 37 & $2.19(1.21-3.96)$ & 0.009 & $1.42(0.75-2.68)$ & 0.28 \\
\hline Absence & 45 & & & & \\
\hline \multicolumn{6}{|c|}{ Tumor number } \\
\hline Multiple & 19 & $2.42(1.27-4.59)$ & 0.007 & $2.70(1.34-5.45)$ & 0.005 \\
\hline Single & 63 & & & & \\
\hline \multicolumn{6}{|c|}{ Tumor size $(\mathrm{cm})$} \\
\hline$>5$ & 25 & $1.25(0.68-2.31)$ & 0.47 & & \\
\hline$\leq 5$ & 57 & & & & \\
\hline \multicolumn{6}{|c|}{ Surgical margin } \\
\hline positive & 12 & $2.29(1.09-4.81)$ & 0.029 & $2.13(0.97-4.72)$ & 0.061 \\
\hline negative & 70 & & & & \\
\hline
\end{tabular}

Table 3. Continued

\begin{tabular}{lrrrrr}
\hline Variable & & Univariate & & Multivariate & \\
& $\mathrm{N}$ & $\mathrm{HR}(95 \% \mathrm{CI})$ & $\mathrm{p}$-value & $\mathrm{HR}(95 \% \mathrm{CI})$ & $\mathrm{p}$-value \\
\hline \multicolumn{2}{l}{ Adjuvant chemotherapy } \\
Yes & 41 & $0.94(0.51-1.72)$ & 0.84 & & \\
No & 41 & & & & \\
\hline
\end{tabular}

$\mathrm{p}=0.006)$; and multiple tumors (HR, 3.34; $95 \% \mathrm{CI}$, $1.69-6.61 ; \mathrm{p}<0.001)$.

Prognostic factors for OS among patients with ICC after hepatic resection

Table 3 shows the prognostic factors for OS. According to the univariate analysis, the CRP/Alb $>0.089$, CA19-9 $>37$, lymph node metastasis, vascular invasion, multiple tumors, and surgical margin positive were significantly associated with OS. The independent prognostic factors for OS, according to the multivariate analysis, were $\mathrm{CRP} /$ Alb ratio $>0.089$ (HR, 3.39; 95\% CI, 1.72-6.67; $\mathrm{p}<0.001$ ); lymph node metastasis (HR, 2.82; 95\%, CI $1.29-6.19 ; \mathrm{p}=0.01)$; and multiple tumors (HR, 2.70; 95\% CI, $1.34-5.45 ; \mathrm{p}=0.005)$.

\section{Discussion}

The current study demonstrated that compared with patients with $\mathrm{CRP} / \mathrm{Alb}$ ratio $\leq 0.089$, those with $\mathrm{CRP} / \mathrm{Alb}$ ratio $>0.089$ had approximately three-fold higher risk for recurrence and death after hepatic resection for ICC. To the best of our knowledge, this was the first report to analyze the relationship between $\mathrm{CRP} / \mathrm{Alb}$ ratio and survival outcomes in patients with ICC.

$\mathrm{CRP} / \mathrm{Alb}$ ratio was considered to be associated with the inflammatory response induced by cancer progression processes, such as tumor growth, invasion, necrosis, hypoxia, or local tissue damage. Previous studies indicated that a high CRP/Alb ratio was associated with large tumor size, tumor depth, microvascular invasion, and lymph node metastasis in gastric cancer, colorectal cancer, and hepatocellular carcinoma (Ishizuka et al., 2016; Shibutani et al., 2016; Saito et al., 2018; Kinoshita et al., 2015). In the current study, CRP/Alb ratio $>0.089$ was significantly associated with a large tumor size and bile duct tumor invasion and tended to be associated with vascular invasion in patients with ICC. The cause of increased poor prognostic risk in patients with $\mathrm{CRP} / \mathrm{Alb}$ ratio $>0.089$ is not clear. However, given the higher frequency of a large tumor size and vascular invasion, cancer progression may contribute to increase the poor prognostic risk in patients with the $\mathrm{CRP} / \mathrm{Alb}>0.089$.

In this study, the serum level of CRP was not associated with the increased risk of recurrence and death. Conversely, Lin et al reported that an elevated preoperative CRP level was associated with poor survival outcomes in patients with ICC (Lin et al., 2016). A possible reason of this discrepancy was the different background inflammatory status of the patients. In the study by Lin et al., (2016) the median tumor size was $5.5 \mathrm{~cm}$ in diameter, bile duct tumor spread was observed in $63 \%$, and the mean CRP level was $1.52 \mathrm{mg} / \mathrm{dL}$. Conversely, in this study, 
the median tumor size was $3.5 \mathrm{~cm}$, bile duct invasion was observed in $48 \%$, and the median CRP level was $0.12 \mathrm{mg} / \mathrm{dL}$. Therefore, the less inflammatory status of the liver in this study population than that in the previous report may have diminished the prognostic impact of CRP elevation alone. Several previous studies indicated that the $\mathrm{CRP} / \mathrm{Alb}$ ratio was a more valuable prognostic marker, compared with other systemic inflammatory markers, such as the modified Glasgow prognostic score (Shibutani et al., 2016; Wei et al., 2015; Kinoshita et al., 2015). Similarly, combining the values of CRP and Alb into a CRP/Alb ratio may be more useful than CRP alone in predicting the survival outcomes of patients with ICC.

Previous studies identified several risk factors for the development of ICC, such as primary sclerosing cholangitis, liver fluke infestation, hepatolithiasis, hepatitis virus, 1,2-dichloropropane and dichlorometane (Bridgewater et al., 2014; Kubo et al., 2014). These risk factors are considered to induce chronic inflammation in the biliary epithelium and potentially lead to cancer development. In this study, viral or alcoholic hepatitis was identified in $42.7 \%$ of all patients with ICC. However, there was no significant difference in the proportion of patients with viral hepatitis or ALT $>30$ between the CRP/ Alb ratio $>0.089$ and $\leq 0.089$ groups. Therefore, CRP/ Alb ratio may not be associated with background liver inflammatory status and carcinogenic potential in liver.

The effective systemic chemotherapy for ICC has not been established because of limited patient numbers in previous reports. Although no standard adjuvant chemotherapy had been adopted in patients with ICC, several reports indicated that gemcitabine or S-1 after surgical resection in patients with biliary tract cancer improved OS (Yoshida et al., 2012; Yamanaka et al., 2014). Moreover, a recent randomized controlled study demonstrated favorable OS in patients with advanced biliary tract cancer after receiving cisplatin plus gemcitabine than gemcitabine alone; in that study, subgroup analysis of patients with ICC showed survival benefit with the combination arm (Valle et al., 2010). Therefore, such regimens of chemotherapy may be possible options for adjuvant treatment in patients with $\mathrm{CRP} /$ Alb ratio $>0.089$.

The current study had some limitations. First, it was retrospective, had a small sample size, and was single-center in design. Second, we decided the cutoff value of CRP/Alb ratio at 0.089 , based on a cutoff finder on small patient number. However, the median cutoff value of CRP/Alb ratio reported in one meta-analysis for the subject of several cancers was 0.095 (Xu et al., 2017). Therefore, we considered the approximated cutoff value in this study as appropriate.

In conclusion, in patients with ICC, the preoperative $\mathrm{CRP} / \mathrm{Alb}$ ratio was an independent predictor of postoperative recurrence and death and may help in the selection of appropriate therapeutic strategies.

\section{Acknowledgements}

This work was supported by the Health, Labour and Welfare Policy Research Grants from the Ministry of
Health, Labour, and Welfare of Japan (Policy Research for Hepatitis Measures [H30-Kansei-Shitei-003]).

\section{Conflict of interest}

No potential conflicts of interest were disclosed.

\section{References}

Bridgewater J, Galle PR, Khan SA, et al (2014). Guidelines for the diagnosis and management of intrahepatic cholangiocarcinoma. J Hepatol, 60, 1268-89

Budczies J, Klauschen F, Sinn BV, et al (2012). Cutoff Finder: a comprehensive and straightforward Web application enabling rapid biomarker cutoff optimization. PLoS One, 7, e51862

Farges O, Fuks D (2010). Clinical presentation and management of intrahepatic cholangiocarcinoma. Gastroenterol Clin Biol, 34, 191-9

Farges O, Fuks D, Le Treut YP, et al (2011). AJCC 7th edition of TNM staging accurately discriminates outcomes of patients with resectable intrahepatic cholangiocarcinoma: By the AFC-IHCC-2009 study group. Cancer, 117, 2170-7.

Haruki K, Shiba H, Shirai Y, et al (2016). The C-reactive protein to albumin ratio predicts long-term outcomes in patients with pancreatic cancer after pancreatic resection. World $J$ Surg, 40, 2254-60

Hashimoto K, Ikeda Y, Korenaga D, et al (2005). The impact of preoperative serum C-reactive protein on the prognosis of patients with hepatocellular carcinoma. Cancer, 103, 1856-64

Ishida S, Hashimoto I, Seike T, et al (2014). Serum albumin levels correlate with inflammation rather than nutrition supply in burns patients: a retrospective study. J Med Invest, 61, 361-8

Ishino Y, Saigusa S, Ohi M, et al (2014). Preoperative C-reactive protein and operative blood loss predict poor prognosis in patients with gastric cancer after laparoscopy-assisted gastrectomy. Asian J Endosc Surg, 7, 287-94

Ishizuka M, Nagata H, Takagi K, et al (2016). Clinical significance of the C-reactive protein to albumin ratio for survival after surgery for colorectal cancer. Ann Surg Oncol, 23, 900-7

Kanda Y (2013). Investigation of the freely available easy-to-use software 'EZR' for medical statistics. Bone Marrow Transplant, 48, 452-8

Kinoshita A, Onoda H, Imai N, et al (2015). The C-reactive protein/albumin ratio, a novel inflammation-based prognostic score, predicts outcomes in patients with hepatocellular carcinoma. Ann Surg Oncol, 22, 803-10

Kubo S, Nakanuma Y, Takemura S, et al (2014). Case series of 17 patients with cholangiocarcinoma among young adult workers of a printing company in Japan. J Hepatobiliary Pancreat Sci, 21, 479-88

Lin ZY, Liang ZX, Zhuang PL, et al (2016). Intrahepatic cholangiocarcinoma prognostic determination using pre-operative serum C-reactive protein levels. BMC Cancer, 16, 792

Liu Z, Jin K, Guo M, et al (2017). Prognostic value of the CRP/ Alb ratio, a novel inflammation-based score in pancreatic cancer. Ann Surg Oncol, 24, 561-8

Liver Cancer Study Group of Japan (2010): General Rules for the Clinical and Pathological Study of Primary Liver Cancer, ed 3. Kanehara \& Co., Ltd., Tokyo

Morris-Stiff G, Gomez D, Prasad KR (2008). C-reactive protein in liver cancer surgery. Eur J Surg Oncol, 34, 727-9

Nakazaki H (1992). Preoperative and postoperative cytokines in patients with cancer. Cancer, 70, 9-13 
Nozoe T, Matsumata T, Kitamura M, et al (1998). Significance of preoperative elevation of serum C-reactive protein as an indicator for prognosis in colorectal cancer. Am J Surg, 176, 335-8

Pan QX, Su ZJ, Zhang JH, et al (2017). Glasgow Prognostic Score predicts prognosis of intrahepatic cholangiocarcinoma. Mol Clin Oncol, 6, 566-74

Pathak S, Nunes QM, Daniels IR, et al (2014). Is C-reactive protein useful in prognostication for colorectal cancer? A systematic review. Colorectal Dis, 16, 769-76

Petrick JL, Braunlin M, Laversanne M, et al (2016). International trends in liver cancer incidence, overall and by histologic subtype, 1978-2007. Int J Cancer, 139, 1534-45

Saito H, Kono Y, Murakami Y, et al (2018). Prognostic significance of the preoperative ratio of C-reactive protein to albumin and neutrophil-lymphocyte ratio in gastric cancer patients. World J Surg, 42, 1819-25

Shibutani M, Maeda K, Nagahara H, et al (2016). Prognostic significance of the preoperative ratio of C-reactive protein to albumin in patients with colorectal cancer. Anticancer Res, 36, 995-1001

Shimada K, Sano T, Sakamoto Y, et al (2007). Surgical outcomes of the mass-forming plus periductal infiltrating types of intrahepatic cholangiocarcinoma: a comparative study with the typical mass-forming type of intrahepatic cholangiocarcinoma. World J Surg, 31, 2016-22

Shirabe K, Mano Y, Taketomi A, et al (2010). Clinicopathological prognostic factors after hepatectomy for patients with mass-forming type intrahepatic cholangiocarcinoma: relevance of the lymphatic invasion index. Ann Surg Oncol, 17, 1816-22

Shrotriya S, Walsh D, Bennani-Baiti N, et al (2015). C-reactive protein is an important biomarker for prognosis tumor recurrence and treatment response in adult solid tumors: A Systematic Review. PLoS One, 10, e0143080

Siegel RL, Miller KD, Jemal A (2015). Cancer statistics. CA Cancer J Clin, 65, 5-29

Spolverato G, Yakoob MY, Kim Y, et al (2015). The impact of surgical margin status on long-term outcome after resection for intrahepatic cholangiocarcinoma. Ann Surg Oncol, 22, 4020-8

Uenishi T, Nagano H, Marubashi S, et al (2014). The long-term outcomes after curative resection for mass-forming intrahepatic cholangiocarcinoma associated with hepatitis C viral infection: a multicenter analysis by Osaka Hepatic Surgery Study Group. J Surg Oncol, 110, 176-81

Uno M, Shimada K, Yamamoto Y, et al (2012). Periductal infiltrating type of intrahepatic cholangiocarcinoma: a rare macroscopic type without any apparent mass. Surg Today, 42, 1189-94

Valle J, Wasan H, Palmer DH, et al (2010). Cisplatin plus gemcitabine versus gemcitabine for biliary tract cancer. $N$ Engl J Med, 362, 1273-81

Wei XL, Wang FH, Zhang DS, et al (2015). A novel inflammation-based prognostic score in esophageal squamous cell carcinoma: the C-reactive protein/albumin ratio. BMC Cancer, 15, 350

Xu HJ, Ma Y, Deng F, et al (2017). The prognostic value of C-reactive protein/albumin ratio in human malignancies: an updated meta-analysis. Onco Targets Ther, 10, 3059-70

Yamamoto Y, Shimada K, Sakamoto Y, et al (2009). Clinicopathological characteristics of intrahepatic cholangiocellular carcinoma presenting intrahepatic bile duct growth. J Surg Oncol, 99, 161-5

Yamanaka K, Hatano E, Kanai M, et al (2014). A single-center analysis of the survival benefits of adjuvant gemcitabine chemotherapy for biliary tract cancer. Int J Clin Oncol,
19, 485-9

Yoshida M, Kudoh J, Homma Y, Kawabe K (2012). New clinical evidence of silodosin, an alpha(1A) selective adrenoceptor antagonist, in the treatment for lower urinary tract symptoms. Int J Urol, 19, 306-16

Zou S, Li J, Zhou H, et al (2014). Mutational landscape of intrahepatic cholangiocarcinoma. Nat Commun, 5, 5696.

This work is licensed under a Creative Commons AttributionNon Commercial 4.0 International License. 